\title{
Pair Distribution Function
}

\section{Simon J. L. Billinge}

Professor of Materials Science and Engineering and Applied Physics and Applied Mathematics

Columbia University

The Pair Distribution Function was subject of a mini-curse. In this presentation it was developed the concepts and fundamentals associated with the theory of Pair Distribution Function. Using didactic examples, the fundamental concepts for minimum understanding were implemented. 